\title{
CT-Guided Oxygen-Ozone Intradiscal Injection Therapy for Thoracolumbar Intervertebral Disc Herniations (Hansen Type II) in Dogs
}

\section{Farkhondeh Fahim Dezhban}

University of Tehran Faculty of Veterinary Medicine

Majid Masoudifard ( $\triangle$ mmfard@ut.ac.ir)

University of Tehran Faculty of Veterinary Medicine https://orcid.org/0000-0001-9087-6919

\section{Mohammad Mehdi Dehghan}

University of Tehran Faculty of Veterinary Medicine

\section{Mohammad Molazem}

University of Tehran Faculty of Veterinary Medicine

\section{Alireza Bahonar}

University of Tehran Faculty of Veterinary Medicine

\section{Amir Ezzati}

University of Tehran Faculty of Veterinary Medicine

Morteza Ezati

University of Tehran Faculty of Veterinary Medicine

\section{Research article}

Keywords: Oxygen-ozone injection, disc shrinkage, intervertebral disc herniation, MRI, dog

Posted Date: October 28th, 2020

DOI: https://doi.org/10.21203/rs.3.rs-97160/v1

License: (9) This work is licensed under a Creative Commons Attribution 4.0 International License.

Read Full License 
1 CT-guided oxygen-ozone intradiscal injection therapy for thoracolumbar intervertebral

2 disc herniations (Hansen Type II) in dogs

3 Farkhondeh Fahim Dezhban $^{1}$, Majid Masoudifard ${ }^{1 *}$, Mohammad Mehdi Dehghan ${ }^{2}$, Mohammad

4 Molazem $^{1}$, Alireza Bahonar ${ }^{3}$, Amir Ezzati ${ }^{4}$ and Morteza Ezati ${ }^{5}$

5

6 Abstract

7 Background: The study aims to investigate the treatment of thoracolumbar herniated intervertebral discs (IVD)

8 in dogs using Oxygen-Ozone $\left(\mathrm{O}_{2}-\mathrm{O}_{3}\right)$ intradiscal injection and assess the results using MRI which achieve more

9 accurate details and procedures.

10 Results: All indices were improved after the treatment, while, three were_statistically significant at $P<0.5$. All 3

11 dogs returned to their normal life after this study.

12 Conclusions: Intradiscal $\mathrm{O}_{2}-\mathrm{O}_{3}$ injection reduces the size of the herniated disc via disc shrinkage and decreasing 13 the pressure of herniated part on the spinal cord.

14 Keywords: Oxygen-ozone injection, disc shrinkage, intervertebral disc herniation, MRI, dog

*Correspondence: mmfard@ut.ac.ir

Full list of authors information is available at the end of the article 


\section{Background}

17 Intervertebral discs are located between al

18 vertebral bodies of the vertebral column,

19 except for the first and second cervical

20 vertebrae (C1 and $\mathrm{C} 2)$, and sacrum.

21 Intervertebral discs possess a complex

22 structure: a thicker fibrous cartilage outer ring

23 (the annulus fibrosus), and a more gelatinous

24 core (the nucleus pulposus). They function as

25 shock absorbers between each vertebra in the

26 vertebral column, allow slight movement of

27 vertebrae, function as ligaments to maintain

28 the vertebrae, and absorb shock to the spine.

29 Dehydration of the nucleus pulposus occurs

30 when it loses water [1]. However, the

31 dehydrated IVDs will no longer be able to

32 function as proper shock absorbers.

33 Intervertebral disc disease (IVDD) usually

34 pushes the nucleus pulposus against the spinal

35 canal and compresses the spinal cord. Effects

36 of IVDD range from bulging to protrusion or

37 extrusion of IVD [2]. A slow aging process in

38 non-chondrodystrophic dogs mainly results in

39 IVD degeneration [3]. If the nucleus pulposus

40 extrudes into the spinal canal and hits the

41 spinal cord (concussion), it will require

42 surgical intervention. More often, it slowly
43 pushes its way into the spinal canal, resulting

44 in pressure over the spinal cord (compression)

$45[2]$.

46 IVDD is diagnosed using a combination of

47 physical and neurological examinations, along

48 with radiographs such as X-rays. In more

49 advanced methods, imaging techniques such as

$50 \mathrm{MRI}$ are obtained from the spine [3].

51 MRI scanners employ strong magnetic

52 fields, magnetic field gradients, and radio

53 waves to generate diagnostic images. It is the

54 best diagnostic tool for the diagnosis of IVD

55 degeneration prior to herniation or extrusion.

56 With the invention of MRI, since it provided

57 more detailed images from the spinal cord,

58 myelography became obsolete [4].

$59 \mathrm{O}_{2}-\mathrm{O}_{3}$ injection for the treatment of disc

60 herniation has been primarily proposed in

61 human medicine. It is now frequently used as a

62 minimally-invasive treatment for IVD

63 herniation. Aside from being a cost-effective

64 procedure, intradiscal injection of this gas

65 demonstrated acceptable clinical results.

66 Along with shrinking the herniated disc, $\mathrm{O}_{2}-\mathrm{O}_{3}$

67 gas possesses anti-inflammatory and analgesic

68 effects on the compressed spinal cord [5].

69 Many studies have confirmed the positive 70 effects of ozone therapy in human medicine [6, 
71 7, 8]. In veterinary medicine, HYUN-JUNG

72 HAN et al. studied the impact of intradiscal

$73 \mathrm{O}_{2}-\mathrm{O}_{3}$ injection on improving IVD herniation.

74 In their study, CT was employed to measure

75 the A-index, which measures the disc

76 herniation area, according to the following

77 formula [5]:

$78 \mathrm{~A}-\operatorname{index}(\%)=\frac{\text { Area of disc herniation } \times 100}{\text { Area of the spinal canal }}$

79 However, IVD is visible in a CT only if the

80 disk is calcified. Therefore, using CT, IVD

81 herniation, and spinal cord compression can be

82 measured with very low accuracy. Therefore,

83 using CT results increases the risk of false

84 smaller measurements on disks that are not
85 shrunk. Moreover, in CT, the difference

86 between a disk and other tissues are not

87 distinguishable [4].

88 To increase accuracy and introduce a

89 diagnostic strategy that poses less radiation on 90 the animal [2], this study was conducted this 91 study using MRI. Meanwhile, multiple indices 92 were measured to evaluate the effect of $\mathrm{O}_{2}-\mathrm{O}_{3}$ 93 therapy on IVDD more accurately.

\section{Results}

95 The quantitative indices for the 13 affected 96 intervertebral discs were measured using Marco

97 PACS software and the mean data values were 98 presented in Table 1.

Table 1 Mean data values before and after intradiscal $\mathrm{O}_{2}-\mathrm{O}_{3}$ injection in 13 IVDs: Astrisks indicates the significant decreasing in MSI and A-index mean data values after treatment.

\begin{tabular}{|c|c|c|c|c|c|c|}
\hline Stage & PG & SS & MSU & MSI & A-Index & B-Index \\
\hline \multicolumn{7}{|l|}{ Before: } \\
\hline Mean & 2.2308 & 1.1538 & 1.1538 & 398.6154 & 12.5708 & 271.5385 \\
\hline $\begin{array}{l}\text { Std. Error of } \\
\text { Mean }\end{array}$ & 0.36080 & 0.22206 & 0.10415 & 39.83726 & 1.80314 & 25.03449 \\
\hline \multicolumn{7}{|l|}{$\begin{array}{l}\text { Std. Deviation } \\
\text { After: }\end{array}$} \\
\hline Mean & 2.6923 & 1.3077 & 1.0000 & $206.6154 *$ & $6.3554 *$ & 253.4615 \\
\hline $\begin{array}{l}\text { Std. Error of } \\
\text { Mean }\end{array}$ & 0.28610 & 0.13323 & 0.16013 & 18.95154 & 1.44976 & 23.57057 \\
\hline \multicolumn{7}{|l|}{$\begin{array}{l}\text { Std. Deviation } \\
\text { Total (bedore and } \\
\text { after): }\end{array}$} \\
\hline Mean & 2.4615 & 1.2308 & 1.0769 & 406.5621 & 9.4631 & 262.5000 \\
\hline & 0.23026 & 0.12779 & 0.09484 & 2.261107 & 1.29269 & 16.94164 \\
\hline $\begin{array}{l}\text { Std. Error of } \\
\text { Mean }\end{array}$ & 1.17408 & 0.65163 & 0.48358 & 8.152538 & 6.59147 & 86.38576 \\
\hline Std. Deviation & & & & & & \\
\hline
\end{tabular}


112 minutes, and was determined according to the

103 neurological examination, radiographic studies, and

113 patient's time of entrance, the process for

1041.5 Tesla MRI. A total of 13 IVDs were injected in 114 anesthesia, involved IVD detection, and final

1053 dogs (from bulging to protrusion). In specific, 8115 injection and removing the spinal needle (for each

106 IVDs were injected in dogs 1 and 3, while 5 IVDs 116 involved IVD). All patients recovered from the

107 were injected in $\operatorname{dog} 2$.

117 procedure normally. Following the injection, some

108 After determining the definite location of the

118 gas contents were seen through surrounding

109 involved IVDs, the dogs were sedated for 119 musculature, and even in the spinal canal. In any

110 intradiscal $\mathrm{O}_{2}-\mathrm{O}_{3}$ injection under $\mathrm{CT}$ scan guidance. 120 case, such incidents generally had no side-effects

111 This intervention lasted approximately 20 to 25121 on recovery or the study in Fig. 1.
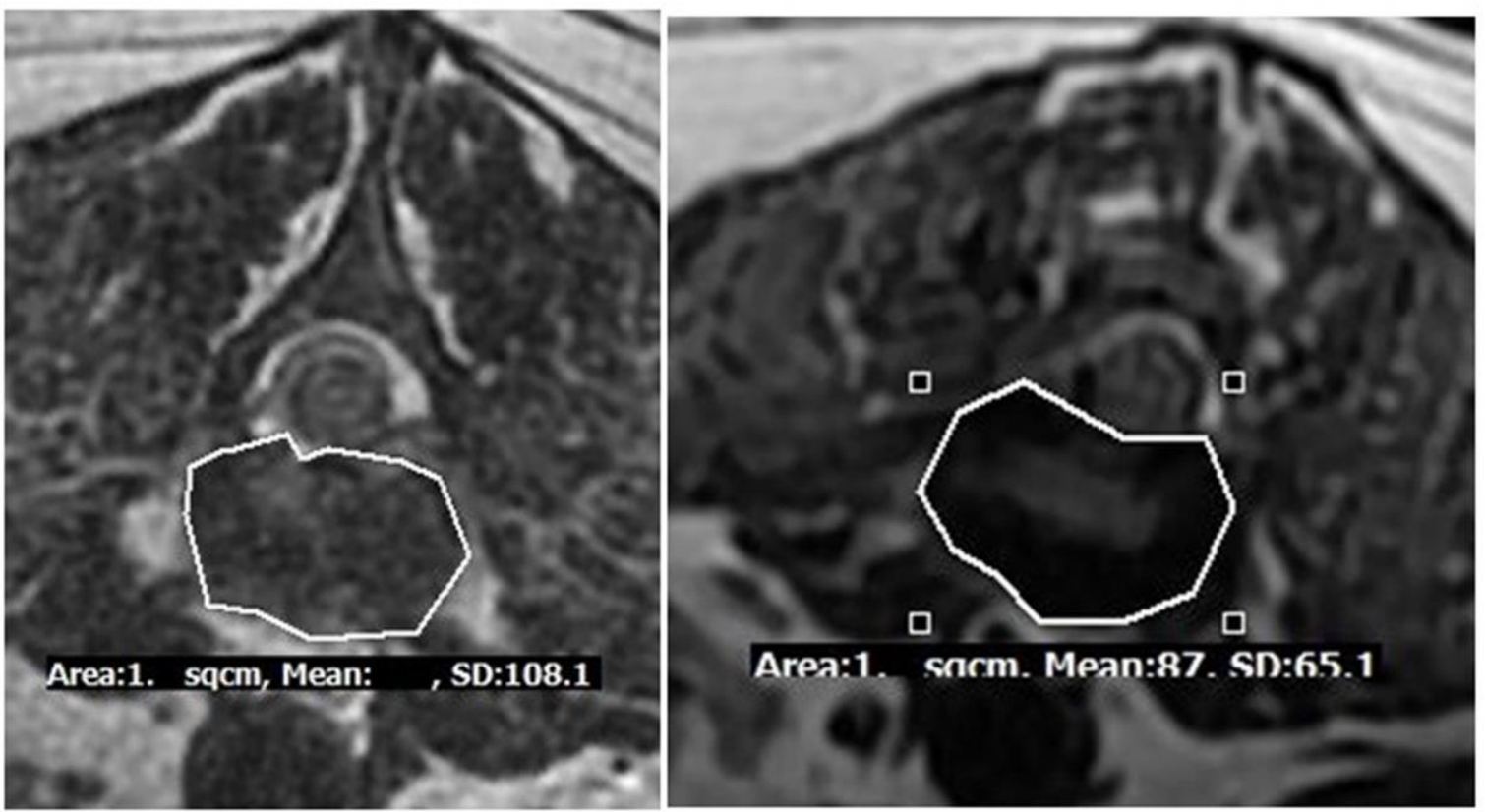

Fig.1 T2 weighted images at the level of L2-L3 from Dog 1 before (1) and after (2) $\mathrm{O}_{2}-\mathrm{O}_{3}$ intradiscal injection, with Bindex and MSI measurement:

R1

$\mathbf{R 2}$ 
125 After 5 weeks from $\mathrm{O}_{2}-\mathrm{O}_{3}$ injection, the clinical

126

127 signs of all three patients were improved. Then, they were reevaluated by 1.5 Tesla MRI, which

128 showed progressive shrinkage of involved IVD in

129 all patients.

Reduction of the involved IVD protrusions,

131 after $\mathrm{O}_{2}-\mathrm{O}_{3}$ injection, was detected on MRI scans

132 taken 5 weeks after the procedure. The results of

133 measured indices were as following:

1. Pfirrmann Grading: this index showed degeneration of IVD contents, except for 3 IVD.

2. Schneiderman Score: this index was not significantly changed.

3. MSU Classification: this index did not experience considerable changes, which may be due to the large grading of this classification. Therefore, there were no significant changes in IVD protrusion status.

4. MSI: this index was changed considerably in all IVDs, up to one-third in some.

5. A-index: this index successfully changed, except in the 2 IVDs, which experienced minor increases.

6. B-index: the variations of this index was interesting, except in the 2 IVDs. In specific, there was a mild increase in the one IVD and no change in only one IVD.
154 The results are shown in Tables 1. They were 155 evaluated using SPSS software and Paired T-test.

\section{Discussion}

157 The present study demonstrates the preliminary 158 quantitative evaluation of IVD size, 5 weeks after 159 intradiscal $\mathrm{O}_{2}-\mathrm{O}_{3}$ injection in dogs. Data confirmed 160 that the degeneration of IVDs occurs following 161 intradiscal $\mathrm{O}_{2}-\mathrm{O}_{3}$ injection (up to $100 \%$ according 162 to MSI index). However, A and B indices were 163 enhanced in $85 \%$ of IVDs, which may need more 164 time due to the chronicity of IVD shrinkage. 165 Previous studies on human subjects demonstrated 166 that IVD shrinkage following intradiscal $\mathrm{O}_{2}-\mathrm{O}_{3}$ 167 injection is influenced by several factors, including 168 the extension of the herniated IVD contents, degree 169 of protrusion, and IVD classification [8].

170 According to HYUN-JUNG HAN et al. [5], 171 comparing our data_indicates that the volume of 172 disc herniation is not related to the disc shrinkage.

173 As the results confirm, degenerative changes 174 were delineated in $77 \%$ of IVDs in the first variant. 175 On the other hand, the $2^{\text {nd }}$ and the $3^{\text {rd }}$ indices were 176 not significantly changed, which can be due to the 177 progressive time-consuming process of IVD 178 degeneration.

179 Pfirrmann and Schneiderman scheme 180 classifications did not experience significant 181 changes. In current study, only one radiologist was 182 responsible for the interpretation of the MR images, 
183 which justifies the possibility of error in the results

184 [11]. To overcome this shortcoming, factors should

185 be evaluated by different radiologists to ensure

186

187 issue regarding these two factors is their detectable

188

189 to alter the MRI intensity of IVD.

190

MSU classification should be evaluated using

191 MRI [12]. As mentioned there, it is measured

192 during a 5-year clinical follow-up on the cases.

193 Therefore, more time is required to evaluate and

194 determine the statistical meaningfulness of this

195 index.

196 To determine and quantify the

197 degeneration, the MSI ( $4^{\text {th }}$ index) was evaluated. A

198

199 treatment, which was statistically meaningful

200 (0.0001, P<0.05). MRI enables assessment of the

201 intensity of the IVD material before and after $\mathrm{O}_{2}-\mathrm{O}_{3}$

202 intradiscal injection.

203 Another valid index to measure the disc

204 shrinkage and decompression of the spinal cord is

205 the $3^{\text {rd }}$ variant (A-index) [5]. In our study, this

206 index was statistically meaningful $(0.006, \mathrm{P}<0.05)$.

207 This variant can be precisely estimated using 1.5

208 Tesla MRI because MRI delineates the accurate

209 margin of the herniated material and compression

210 of spinal cord parenchyma.

211 The last variant (B-index) is measured to 212 evaluate the IVD shrinkage during the 5-week
213 experiment period. The measured B-index value

214 was also statistically valid, which was measured up 215 to $0.003(\mathrm{P}<0.05)$. In addition, this variant was 216 measured by MRI, which provides a gold standard 217 of IVD diagnosis (due to the cartilaginous structure 218 of the IVD).

219 According to A and B indices, we concluded 220 that the IVD shrinkage occurred gradually in 5 221 weeks. On the other hand, according to HYUN222 JUNG HAN et al. [5], there is no relationship 223 between disc shrinkage and the volume of disc 224 herniation. However, more time is needed to 225 evaluate other factors (due to Pfirrmann, 226 Schneiderman, and MSU classification data in our 227 study). Therefore, intradiscal ozone-oxygen 228 injection yields progressive decompressive and 229 anti-inflammatory effects on the spinal cord. 230 Moreover, it has preventive influences on 231 deterioration or recurrence of clinical signs. 232 Nevertheless, more time is required to compare the 233 results with laminectomy or fenestration [2].

234 We also introduced intradiscal $\mathrm{O}_{2}-\mathrm{O}_{3}$ injection 235 as an alternative therapy for herniated IVDD with 236 low invasion, long-lasting effects. Moreover, 237 compared with glucocorticoid therapeutics and 238 surgery, this method is a cost-effective solution.

239 However, despite being useful in chronic patients, 240 intradiscal injection of $\mathrm{O}_{2}-\mathrm{O}_{3}$ is not a desirable 241 therapy in patients that require rapid 242 decompression. In specific, it is beneficial in 
243 patients who demonstrate clinical improvements by

244

245 relapses. All the dogs included in this study had

246 chronic problems and did not require emergency

247 surgery. Samples with no deep pain perception in

248 the clinical examination were excluded since they

249 need emergency operation to decompress the spine.

The density of the involved disc is decreased

251 after $\mathrm{O}_{2}-\mathrm{O}_{3}$ intradiscal injection. This intradiscal

252 shrinkage creates free space for the compressed

253 spine to move regardless of herniated disc materials

254 in the spinal cord. This confirmed by MRI data and

255 clinical signs. Disc shrinkage also guaranteed that

256 the treated IVD would not remain herniated or

257 extruded, and thus, the possibility of recurrence of

258 pain and clinical signs were reduced considerably

259 [5]. Therefore, the $\mathrm{O}_{2}-\mathrm{O}_{3}$ therapy offers a

260 replacement for previous conservative methods,

261 which may prevent further extrusion or recurrence

262 of herniation of IVD [14].

263 There are other advantages in using the $\mathrm{O}_{2}-\mathrm{O}_{3}$

264 mixture as a non-harmful gas with analgesic and

265 anti-inflammatory effects, even when this gas is in

266 direct contact with the spinal cord. The anti-

267 inflammatory feature of the ozone-oxygen mixture

268 functions via inhibiting inflammatory inducers and

269 pain-producing mediators such as prostaglandins

270 [15]. Moreover, due to its strong bactericidal

271 activity, it has fewer complications, which
272 considerably decreases the risk of abscess

273 formation from skin contamination [16, 17].

274 The other noticeable advantage of this method 275 is its simplicity and short anesthesia duration, 276 which plays an essential role in these patients. In 277 addition, it requires minimal hospitalization 278 (approximately 24 hours) for post-surgical checks 279 of the side-effects. In laminectomy and other 280 surgical methods, hospitalization time is 281 considerably more. Furthermore, the cost of this 282 procedure is significantly lower than surgery or 283 other alternatives, specifically in the long-term, as 284 this method prevents further problems with the 285 injected IVD.

\section{Conclusions}

287 In this study, a recognizable disc shrinkage was 288 confirmed in 1.5 Tesla MRI. However, the degree 289 of this shrinkage requires more time and is related 290 to the extent of herniated disc material 291 degeneration. Nevertheless, no specific 292 complications were detected. In conclusion, it is 293 observed that intradiscal $\mathrm{O}_{2}-\mathrm{O}_{3}$ mixture injection 294 under CT scan guidance can be helpful, and 295 minimally invasive, in the decompression of disc 296 herniations.

\section{Methods}

298 Animals: Among the dogs admitted to the Small 299 Animal Teaching Hospital (Faculty of Veterinary 300 Medicine, University of Tehran), 3 dogs with 
301 various types of neurologic abnormalities were

302

303 request. The animals had clinical signs related to

304 thoracolumbar IVDD and positive deep pain

305 perception (DPP). In addition, dogs under 8 year-

306 old with no clinical signs relevant to thoracolumbar

307 IVDD, along with samples with other major

308 diseases such as cardiac or endocrinopathy, were

309 excluded.

310 Samples 1 and 3 were female, while sample

311 number 2 was male, and were 12, 10, and 9 years

312 old, respectively. The patients demonstrated long-

313 lasting periodic problems in gaining weight. Their

314 body weight ranged from 7 to $28 \mathrm{Kgs}$. The patients

315 were gone through complete neurological

316 examination by an experienced surgeon and were

317 diagnosed with thoracolumbar intervertebral disc

318 herniation (IVDH) on physical, neurological,

319 diagnostic imaging views (radiography and MRI),

320 complete blood count profile, and serum

321 biochemical analysis. Different ranges of disc

322 herniation were detected in thirteen intervertebral

323 joint spaces. Deep pain perception (DPP) was

324 positive in all patients, and they had no other

325 systemic diseases. However, they had difficulty in

326 jumping and running.

327 On the contrary, none had urinary or fecal

328 incontinence. Sample 1 had delayed right hind limb

329 paw replacement and hopping test, while samples 2359

330 and 3 both had bilateral delayed paw replacement 360 was used to evaluate the degenerative 


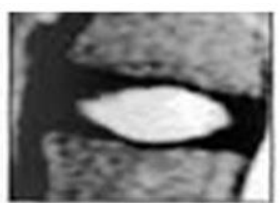

Grade I

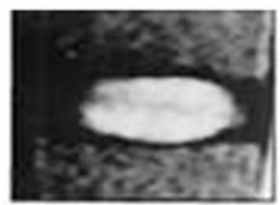

Grade II

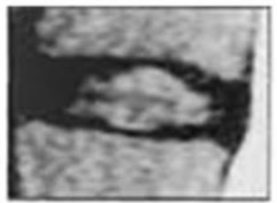

Grade III

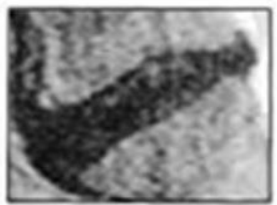

Grade IV

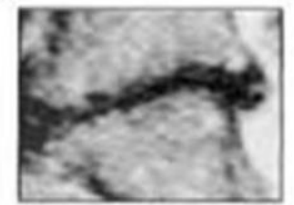

Grade V

A)

B)

C)

D)

E)

\begin{abstract}
A) Grade I = Normal disc intensity
B) Grade II = Mild hypointensities through nucleus polposus

C) Grade III = Increasing hypointensities through nucleus polposus associated with mild decreasing in IVD height

D) Grade IV: Decreasing in IVD height without differentiation of nucleus polposus and annulus fibrosus; totally hypointense

E) Grade $\mathrm{V}=$ Severe decreasing in IVD height without any differentiation of nucleus polposus and annulus fibrosus; it is totally hypointense
\end{abstract}

Fig. 2 T2 Weighted image which indicates Pfirrmann Grading

2) Schneiderman classification scheme: This 374 scheme evaluates the degenerative 375 changes in height and signal intensity of 376 the intervertebral IVDH discs in T2W 377 images, before and 5 weeks after 378 intradiscal injection in Table 1 [11].
3) MSU index: MSU (Michigan State University) index indicates the size of disc herniation and its location in the spinal canal. Moreover, it assesses the degree of disc herniation before and 5 weeks after intradiscal injection. It was introduced by the MSU classification system and is 


\begin{tabular}{ccccc}
\hline Grade & $\mathbf{0}$ & $\mathbf{1}$ & $\mathbf{2}$ & $\mathbf{3}$ \\
\hline $\begin{array}{c}\text { Intervertebral disc } \\
\text { intensity }\end{array}$ & Normal height of & Speckled pattern or & Diffuse loss of & Signal void in IVD \\
& IVD & Neterogeneous with & signal in nucleus \\
polposus and & intensity \\
& $\begin{array}{c}\text { intensity in annulus } \\
\text { fibrosis and nucleus } \\
\text { polposus }\end{array}$ & in nucleus polposus & annulus fibrosus & in IVD height \\
& & & \\
\hline
\end{tabular}

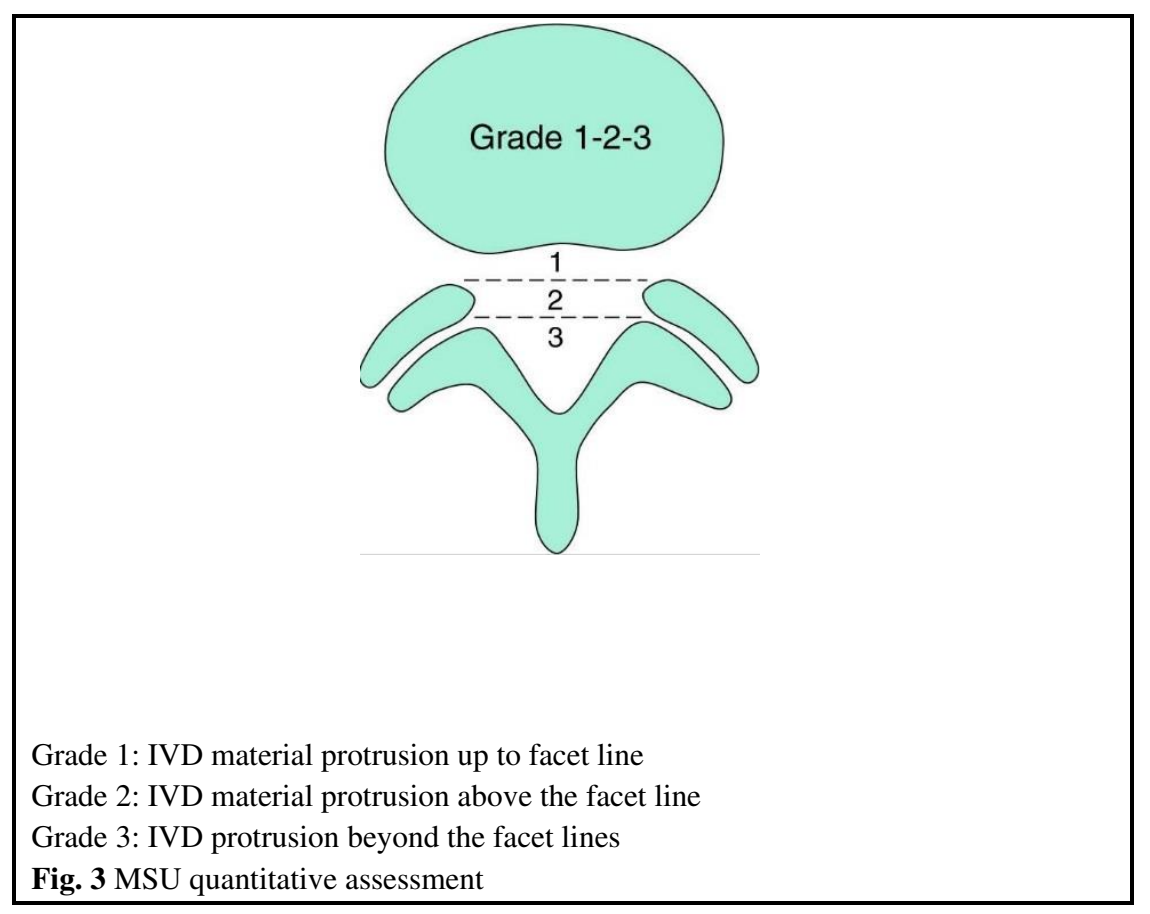

4) MSI: Evaluation and comparison of the 402

Mean Signal Intensity (MSI) of the IVD 404

were performed before and 5 weeks after

intradiscal injection [13].

406

5) A-index: Measurement of the disc herniation area, in comparison with the spinal canal area, was performed before and 5 weeks after intradiscal injection, as proposed in Fig. 1 [1].

407

6) B-index: Measurement and comparison of herniated intervertebral disc area, before and 5 weeks after intradiscal injection, was performed using the following equation:

$$
B-\operatorname{index}(\%)=
$$

Intervertebral disc surface area before ozone-oxygen injection $\times 100$ Intervertebral disc surface area after 5 weeks after ozone-oxygen injection

409 The injection sites in the dorsal thoracolumbar

410 area were first marked by the CT guide and were

411 then surgically scrubbed (See in Fig. 4). 


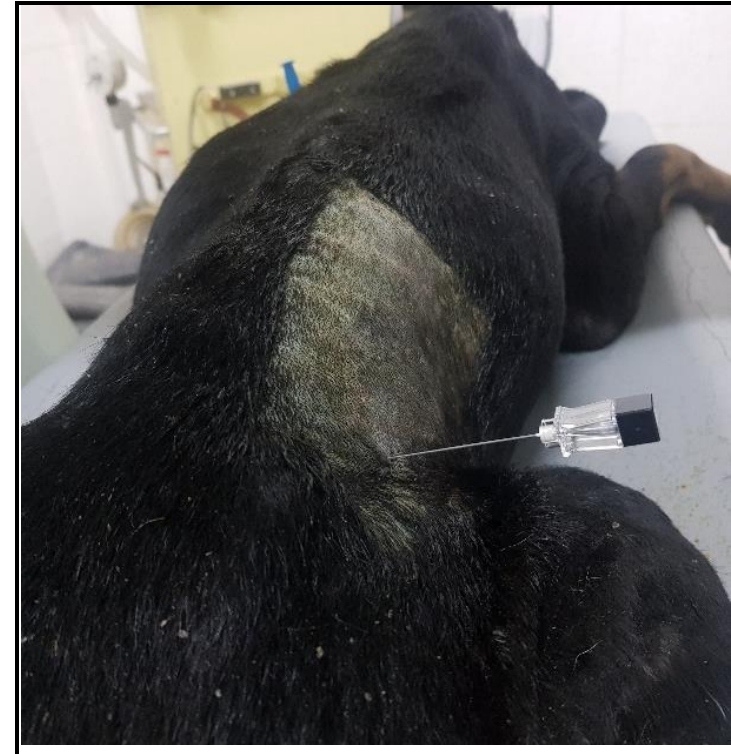

Fig. 4 Preparation of the patient and injection of the spinal needle in the L4-L5 IVD under CT scan guidance

414 The skin was perforated using a 2.5 inch $22 \mathrm{G}$

415 spinal needle, and the needle was guided through

416 epaxial muscles to the center of the herniated disc

417 from the lateroventral side of the articular facet

418 (See in Fig. 5).

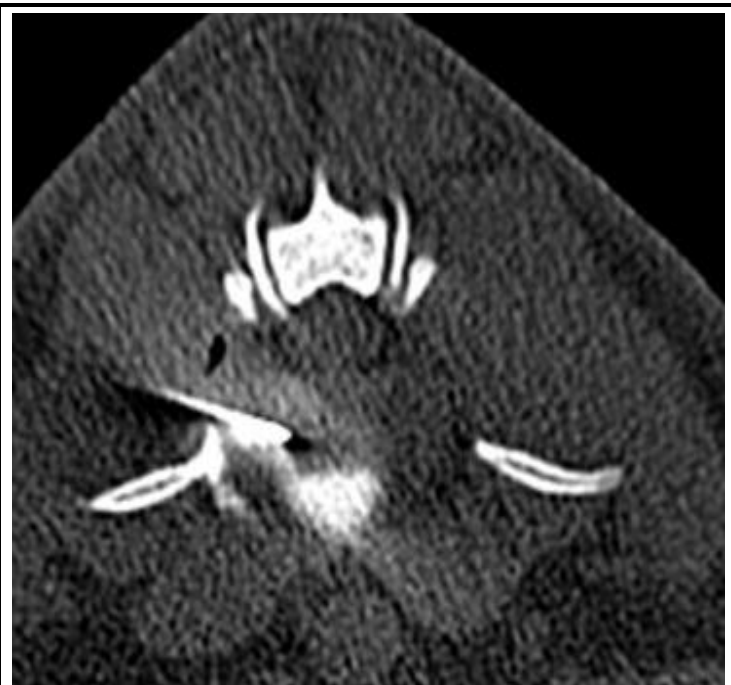

Fig. $5 \cdot \mathrm{O}_{2}-\mathrm{O}_{3}$ intradiscal injection under $\mathrm{CT}$ guidance Red arrow: Spinal needle in L4-L5 IVD

Yellow arrows: $\mathrm{O}_{2}-\mathrm{O}_{3}$ gas bubbles injection 439 Hospital, Faculty of Veterinary Medicine of University 440 of Tehran. The Veterinary Teaching Hospital supported

441 Ms. Fahim Dezhban for using radiology and CT scan 442 equipments.

\section{Authors' contributions}

444 All authors have read and approved the final manuscript.

\section{Funding: not applicable}

\section{Availability of data and materials: yes}

\section{7}

Ethics approval and consent to participate: yes from

448 faculty of veterinary of university of Tehran 
4521 Department of Veterinary Radiology, Faculty of

453 Veterinary Medicine, University of Tehran, Tehran, Iran.

4542 Department of Veterinary Surgery, Faculty of

455 Veterinary Medicine, University of Tehran, Tehran, Iran.

4563 Department of Veterinary Epidemiology, Faculty of

457 Veterinary Medicine, University of Tehran, Tehran, Iran.

$458{ }^{4}$ Infectious diseases specialist, General hospital, Tehran,

459 Iran. ${ }^{5}$ Department of Small Animal Internal Medicine,

460 Faculty of Veterinary Medicine, University of Tehran,

461 Tehran, Iran.

1. Andreula C. Ozone therapy. Neuroradiology. 2011; 53: 207.

2. Berent AC, Tobias KM. Hepatic vascular anomalies. Veterinary Surgery Small Animal. 2012; 1: 1624-1658.

3. Brisson BA. Intervertebral disc disease in dogs. Veterinary Clinics of North America: Small Animal Practice. 2010; 40: 829-858.

4. Robertson IAN, Thrall D E. Imaging dogs with suspected disc herniation: pros and cons of myelography, computed tomography, and magnetic resonance. Veterinary radiology \& ultrasound. 2011; 52: S81-S84.

5. Han HJ, Kim JY, Jang HY, Lee B, Yoon JH, Jang SK, Jeong SW. Fluoroscopicguided intradiscal oxygen-ozone injection therapy for thoracolumbar intervertebral disc herniations in dogs. in vivo. 2007; 21 : 609-613.
6. Buric J, Rigobello L, Hooper D. Five and ten year follow-up on intradiscal ozone injection for disc herniation. International journal of spine surgery. $2014 ; 8$.

7. ZhangY, Ma Y, Jiang J, Ding T, Wang J. Treatment of the lumbar disc herniation with intradiscal and intraforaminal injection of oxygen-ozone. Journal of back and musculoskeletal rehabilitation. 2013; 26: $317-322$
8. Lehnert T, Naguib NN, Wutzler S, NourEldin NEA, Bauer RW, Kerl JM, Balzer JO. Analysis of disk volume before and after CT-guided intradiscal and periganglionic ozone-oxygen injection for the treatment of lumbar disk herniation. Journal of Vascular and Interventional Radiology. 2012; 23:1430-1436.

9. Mwale F, Iatridis JC, Antoniou J. Quantitative MRI as a diagnostic tool of intervertebral disc matrix composition and integrity. European Spine Journal. 2008; 17: 432

10. Grunert P, Hudson KD, Macielak MR, Aronowitz E, Borde BH, Alimi M., Härtl R. Assessment of intervertebral disc degeneration based on quantitative MRI analysis: an in vivo study. 2014; 39: E369.

11. Nagy SA, Juhasz I, Komaromy H, Pozsar K, Zsigmond I, Perlaki G, Bogner P. A 
statistical model for intervertebral disc

degeneration: determination of the optimal

t2 cut-off values. Clinical neuroradiology. 2014; 24: 355-363.

12. Mysliwiec LW, Cholewicki J, Winkelpleck MD, Eis GP. MSU classification for herniated lumbar discs on MRI: toward developing objective criteria for surgical selection. European spine journal. 2010. 19:1087-1093.

13. Niemeläinen R, Videman $T$, Dhillon SS, Battié MC. Quantitative measurement of intervertebral disc signal using MRI. Clinical radiology. 2008; 63: 252-255.

14. Andreula CF, Simonetti L, De Santis F, Agati R, Ricci R, Leonardi M.
Minimally invasive oxygen-ozone therapy for lumbar disk herniation. American Journal of Neuroradiology. 2003; 24: 996-1000.

15. Bonetti M, Fontana A, Cotticelli B, Dalla Volta G, Guindani M, Leonardi M. Intraforaminal $\mathrm{O} 2-\mathrm{O} 3$ versus periradicular steroidal infiltrations in lower back pain: randomized controlled study. American journal of neuroradiology. 2005; 26: 996-1000.

16. Rilling S, Viebahn R. The use of ozone in medicine. KF Haug Publishers; Portland, OR: US distributor, Medicina Biologica. 1987. 17. Viebahn-Haensler R, Lee A. The use of ozone in medicine. 2002; (pp. 421). Heidelberg: ODREIPublishers. 


\section{Figures}

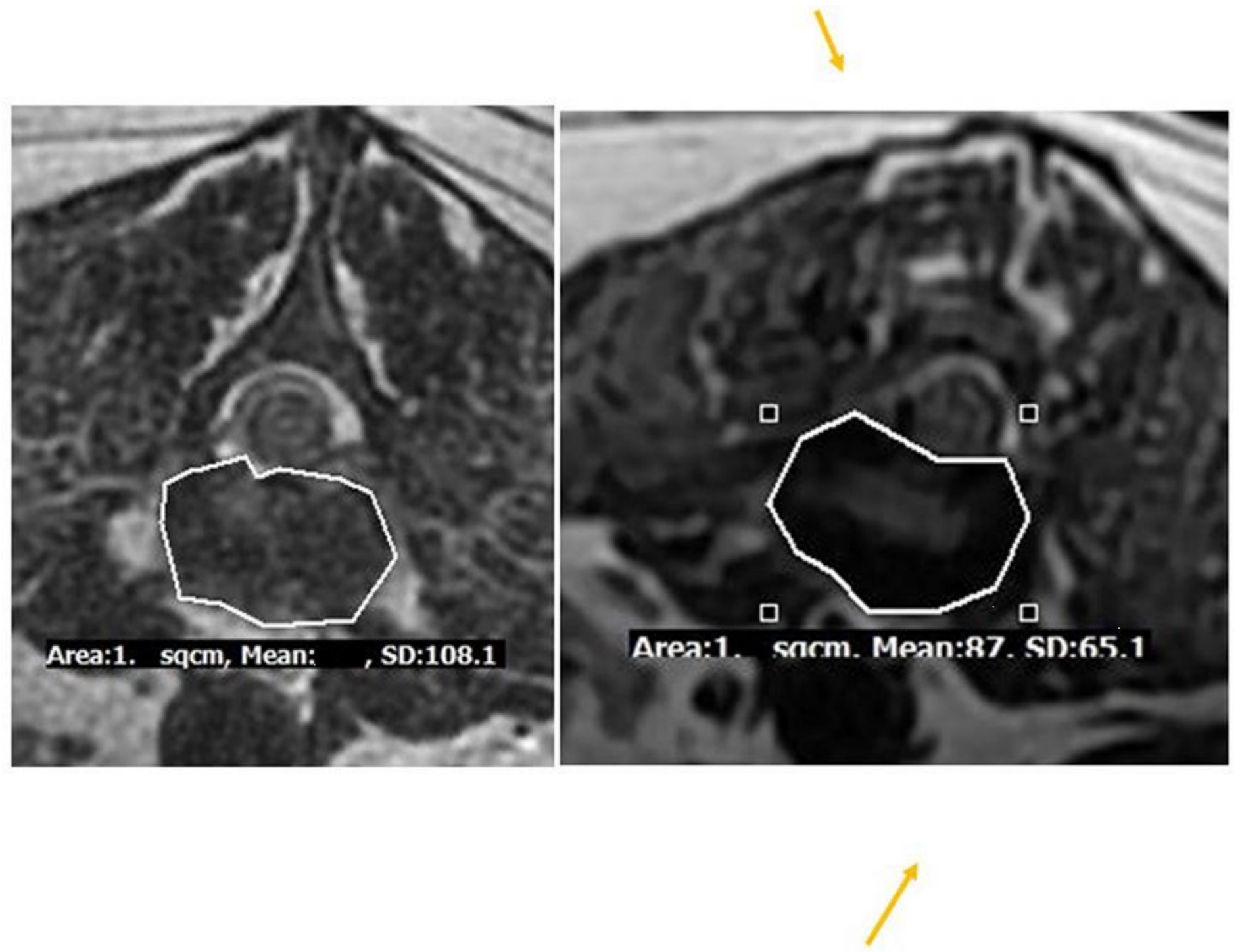

Figure 1

1 T2 weighted images at the level of L2-L3 from Dog 1 before (1) and after (2) 02-03 intradiscal injection, with B-index and MSI measurement: R1 R2 


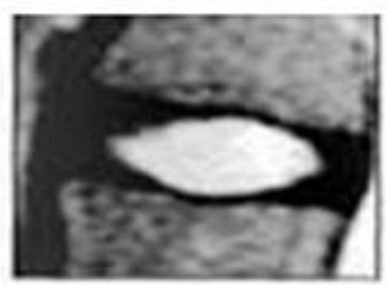

Grade I

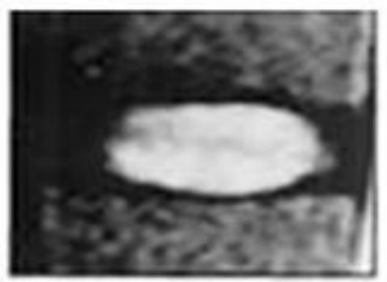

Grade II

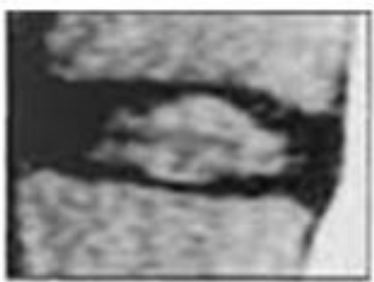

Grade III

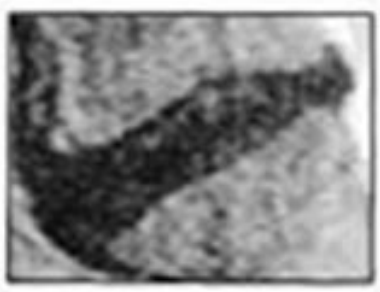

Grade IV

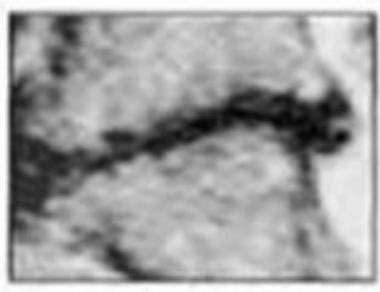

Grade V
A)

D)
C)

E)

A) Grade I = Normal disc intensity

B) Grade II = Mild hypointensities through nucleus polposus

C) Grade III = Increasing hypointensities through nucleus polposus associated with mild decreasing in IVD height

D) Grade IV: Decreasing in IVD height without differentiation of nucleus polposus and annulus fibrosus; totally hypointense

E) Grade V = Severe decreasing in IVD height without any differentiation of nucleus polposus and annulus fibrosus; it is totally hypointense

Figure 2

T2 Weighted image which indicates Pfirrmann Grading 


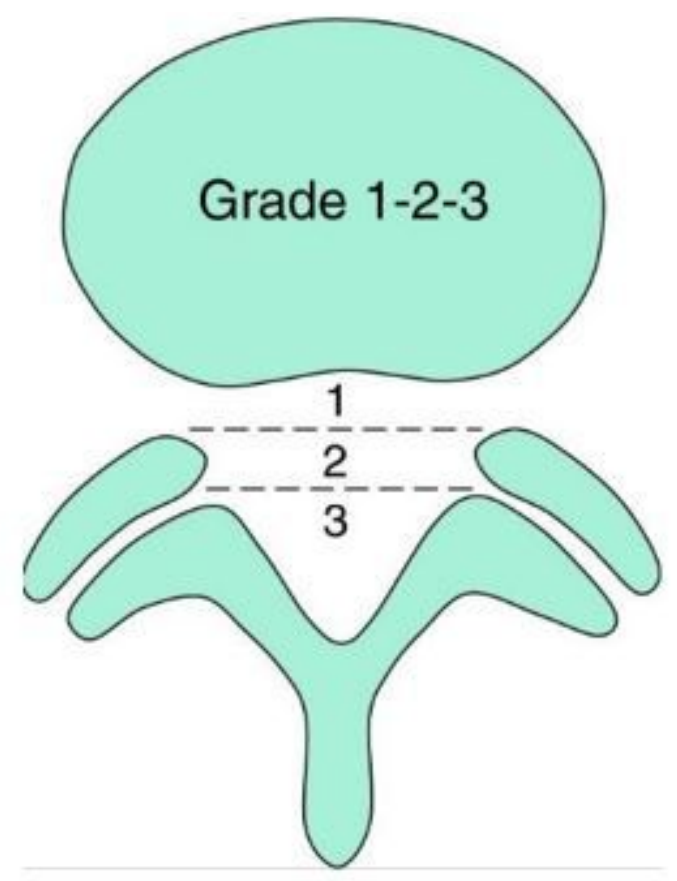

Figure 3

MSU quantitative assessment Grade 1: IVD material protrusion up to facet line Grade 2: IVD material protrusion above the facet line Grade 3: IVD protrusion beyond the facet lines 


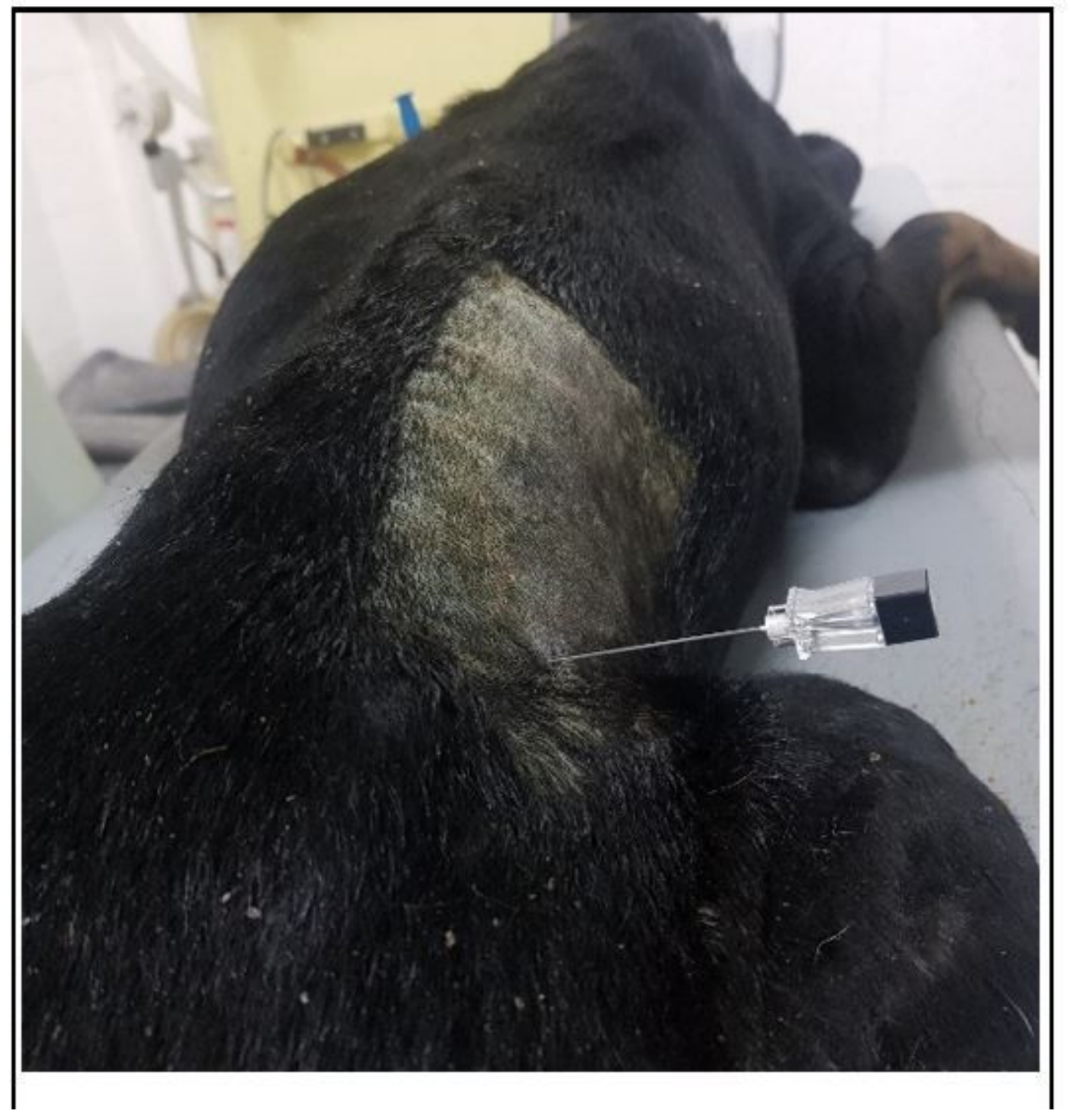

Figure 4

Preparation of the patient and injection of the spinal needle in the L4-L5 IVD under CT scan guidance 


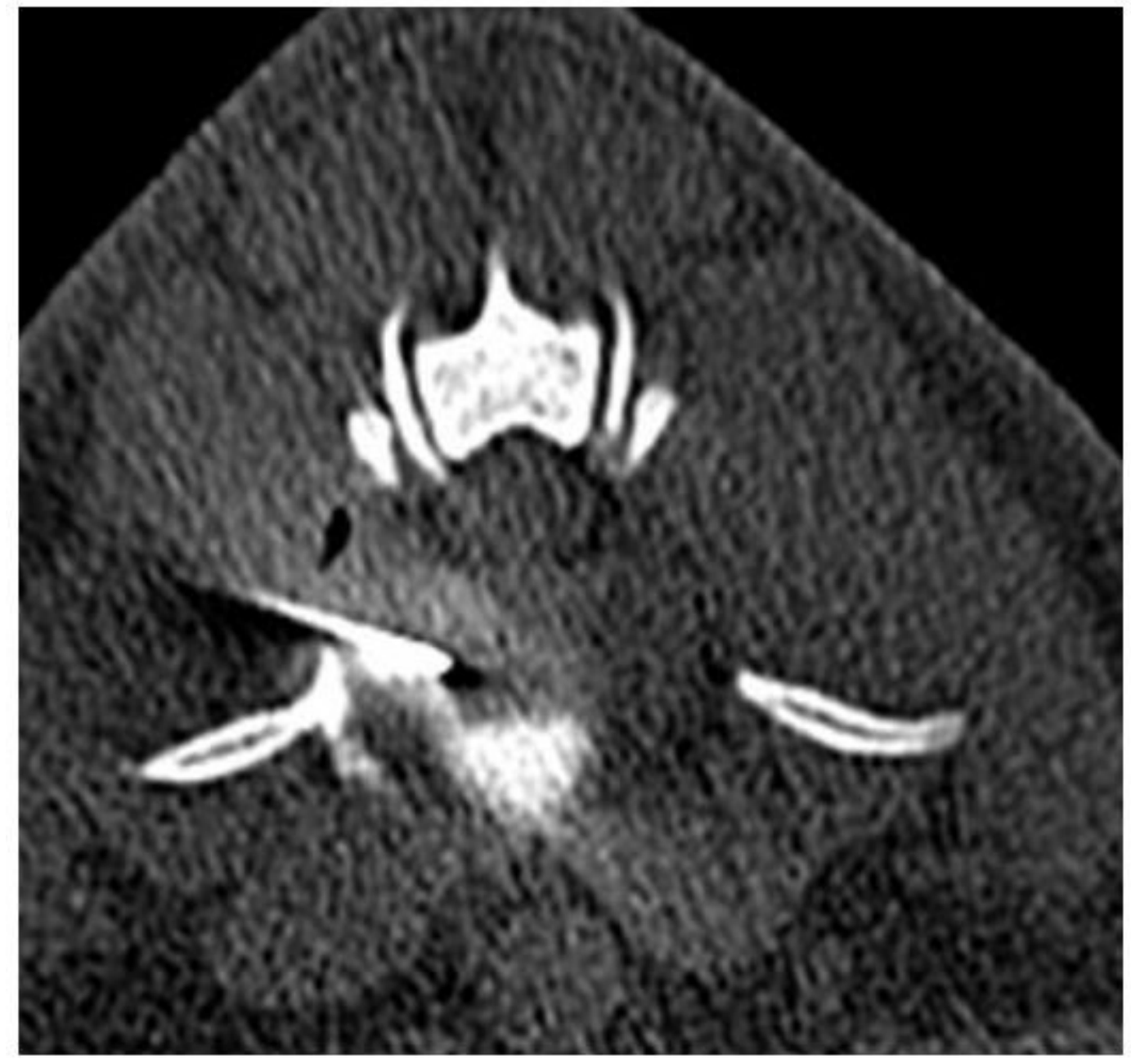

\section{Figure 5}

02-03 intradiscal injection under CT guidance Red arrow: Spinal needle in L4-L5 IVD Yellow arrows: 02O3 gas bubbles injection 